\title{
FRACTIONAL COMPLEX TRANSFORM FOR FRACTIONAL DIFFERENTIAL EQUATIONS
}

\author{
Zheng-Biao $\mathrm{Li}^{1}$, Ji-Huan $\mathrm{He}^{*^{2}}$
}

1. College of Mathematics and Information Science, Qujing Normal University, Qujing, Yunnan 655011, China

2. National Engineering Laboratory of Modern Silk, Soochow University,

No. 1, Shizi Street, Suzhou 215006, China

*Corresponding author: J.H. He, email: Jhhe@dhu.edu.cn

\begin{abstract}
Fractional complex transform is proposed to convert fractional differential equations into ordinary differential equations, so that all analytical methods devoted to advanced calculus can be easily applied to fractional calculus. Two examples are given.
\end{abstract}

Keywords: Modified Riemann-Liouville Derivative, Fractional Differential Equation, Exact Solution

\section{INTRODUCTION}

During the past decades, fractional differential equations appeared more and more frequently in different research areas. Many important phenomena in electromagnetics, acoustics, viscoelasticity, electrochemistry, cosmology and material science can be better described by fractional differential equations. Consequently, considerable attention has been given to the solution of the fractional differential equations. Many powerful methods for solving fractional differential equations were appeared in open literature, such as the variational iteration method [1,2], the homotopy perturbation method [3] and the expfunction method[4]. Although these methods lead to exact solutions in some special cases, exact solutions are much needed in engineering applications. In this paper we will suggest a novel and universal approach to the search for exact solutions of fractional equations, we name the method as "fractional complex transform".

\section{FRACTIONAL COMPLEX TRANSFORM}

In order to illustrate the basic idea of the suggested method, we consider first the following fractional differential equation [5]:

$$
\partial_{t}^{\alpha} u(x, t)=k \partial_{x}^{\beta} u(x, t), \mathrm{t} \in \mathrm{R}^{+}, \mathrm{x} \in \mathrm{R},
$$

which subject to the initial condition

$$
u(x, 0)=u_{0}(x)=2 x,
$$

where $k$ is a positive coefficient, $0<\alpha<1,0<\beta<1, u(x, t)$ is the real-valued variable, $\partial_{t}^{\alpha}$ and $\partial_{x}^{\beta}$ are modified Riemann-Liouville fractional derivatives. 
The fractional complex transform is very simple and straightforward. Introducing a complex variable $\xi$ defined as

$$
\xi=\frac{p x^{\beta}}{\Gamma(1+\beta)}+\frac{q t^{\alpha}}{\Gamma(1+\alpha)} .
$$

where $p, q$ are constants which are unknown to be further determined .

Substituting Eq.(3) into Eq.(1), we obtain

$$
(k p-q) u_{\xi}=0 .
$$

Case 1. $k p-q \neq 0$.

Under such case, the solution is

$$
u(\xi)=c,
$$

where $c$ is a constant. It is a trivial solution without satisfying the initial condition, Eq.(2).

Case 2. $k p-q=0$.

Considering the initial condition, we choose

$$
u_{\xi}=\frac{2}{\beta}\left(\frac{\Gamma(1+\beta)}{p}\right)^{\frac{1}{\beta}}{ }_{\xi}^{\frac{1}{\beta}-1} \text {. }
$$

or

$$
u(\xi)=2\left(\frac{\Gamma(1+\beta)}{p}\right)^{\frac{1}{\beta}} \xi^{\frac{1}{\beta}} .
$$

which satisfies the initial condition.

Substituting Eq.(3) into Eq.(7) results in an exact solution satisfying the initial condition:

$$
u(x, t)=2\left(\frac{\Gamma(1+\beta)}{p}\right)^{\frac{1}{\beta}}\left(\frac{p x^{\beta}}{\Gamma(1+\beta)}+\frac{q t^{\alpha}}{\Gamma(1+\alpha)}\right)^{\frac{1}{\beta}} .
$$

where $p$ and $q$ are free parameters satisfying $k p-q=0$.

\section{AN EXAMPLE}

We now consider the fractional wave equation [5]:

$$
\partial_{t}^{2 \alpha} u(x, t)=k^{2} \partial_{x}^{2 \beta} u(x, t), \mathrm{x} \in[a, b], \mathrm{t} \in \mathrm{R}^{+}, \mathrm{x} \in \mathrm{R},
$$

with the following initial condition

$$
u(x, 0)=u_{0}(x)=2 x^{2}+x,
$$

where $k$ is a positive coefficient, $0<2 \alpha<1,1<2 \beta<2, \quad u(x, t)$ is the real-valued variable, $\partial_{t}^{\alpha}$ and $\partial_{x}^{\beta}$ are modified Riemann-Liouville fractional derivatives.

By the fractional complex transform, Eq.(3), Eq.(9) reduces to

$$
\left(q^{2}-k^{2} p^{2}\right) u_{\xi \xi}=0 \text {. }
$$

Case 1. $q^{2}-k^{2} p^{2} \neq 0$.

From Eq. (11), we have 
where $c_{1}$ and $c_{2}$ are constants.

$$
u(\xi)=c_{1} \xi+c_{2}
$$

We, therefore, obtain the following solution

$$
u(x, t)=c_{1}\left(\frac{p x^{\beta}}{\Gamma(1+\beta)}+\frac{q t^{\alpha}}{\Gamma(1+\alpha)}\right)+c_{2} .
$$

This is also trivial solution without any physical insights.

Case 2. $q^{2}-k^{2} p^{2}=0$.

Considering the initial condition, we have

$$
u_{\xi \xi}=2\left(\frac{\Gamma(1+\beta)}{p}\right)^{\frac{2}{\beta}} \frac{2}{\beta}\left(\frac{2}{\beta}-1\right) \xi^{\frac{2}{\beta}-2}+\left(\frac{\Gamma(1+\beta)}{p}\right)^{\frac{1}{\beta}} \frac{1}{\beta}\left(\frac{1}{\beta}-1\right) \xi^{\frac{1}{\beta}-2} .
$$

Integrating twice yields

$$
u(\xi)=2\left(\frac{\Gamma(1+\beta)}{p}\right)^{\frac{2}{\beta}} \xi^{\frac{2}{\beta}}+\left(\frac{\Gamma(1+\beta)}{p}\right)^{\frac{1}{\beta}} \xi^{\frac{1}{\beta}}+c,
$$

where $c$ are an integral constant.

We, therefore, obtain the following solution:

$u(x, t)=2\left(\frac{\Gamma(1+\beta)}{p}\right)^{\frac{2}{\beta}}\left(\frac{p x}{\Gamma(1+\beta)}+\frac{q t^{\alpha}}{\Gamma(1+\alpha)}\right)^{\frac{2}{\beta}}+\left(\frac{\Gamma(1+\beta)}{p}\right)^{\frac{1}{\beta}}\left(\frac{p x}{\Gamma(1+\beta)}+\frac{q t^{\alpha}}{\Gamma(1+\alpha)}\right)^{\frac{1}{\beta}}+c$,

where $c, p$ and $q$ are free parameters under the constraint,$q^{2}-k^{2} p^{2}=0$.

Incorporating the initial condition, Eq.(10), the parameters in Eq.(16) can be identified as follows

$$
c=0, p=\Gamma(1+\beta) \text { and } q=k \Gamma(1+\beta) \text {. }
$$

The exact solution is obtained, which reads

$$
u(x, t)=2\left(x^{\beta}+\frac{k \Gamma(1+\beta) t^{\alpha}}{\Gamma(1+\alpha)}\right)^{\frac{2}{\beta}}+\left(x^{\beta}+\frac{k \Gamma(1+\beta) t^{\alpha}}{\Gamma(1+\alpha)}\right)^{\frac{1}{\beta}} .
$$

\section{CONCLUSION}

A new and universal approach, the fractional complex transform, is suggested in this paper to find exact solutions of fractional equations. The solution procedure is simple and might be found wide applications in engineering.

\section{ACKNOWLEDGEMENTS}

The work is supported by NSF11061028, and the Science Research Foundation of Yunnan Educational Department under Grant No. 08Y0302, and the Science Research Foundation of Qujing Normal University Grant No. 2008MS018. 


\section{REFERENCES}

[1] J. H. He, G.C. Wu, F. Austin, The Variational Iteration Method Which Should Be Followed, Nonlinear Science Letters A, 1(2010) 1-30

[2] G.C. Wu \& E.W.M. Lee. Fractional variational iteration method and its application Physics Letters A, 374 (2010) 2506-2509

[3] A. Golbabai, K. Sayevand, The Homotopy Perturbation Method for Multi-order Time Fractional Differential Equations , Nonlinear Science Letters A, 1(2010) 147154

[4] S. Zhang, et al., A generalized Exp-function method for fractional Riccati differential equations, Communications in Fractional Calculus, 1(2010)48-51

[5] G. Jumarie, Fractional partial differential equations and modified RiemannLiouville derivative new methods for solution, J. Appl. Math. \& Computing 1(2007)31-48 